\title{
Large-vessel vasculitis following the Pfizer-BioNTech COVID-19 vaccine
}

\author{
Michele Gilio ${ }^{1}\left[\right.$. Giulio De Stefano ${ }^{1,2}$
}

Received: 23 September 2021 / Accepted: 8 December 2021 / Published online: 24 January 2022

(c) The Author(s), under exclusive licence to Società Italiana di Medicina Interna (SIMI) 2021

\section{Dear Sir,}

At the end of 2019, a novel coronavirus now known as severe acute respiratory syndrome coronavirus 2 (SARS$\mathrm{CoV}-2$ ) was identified as the cause of a cluster of pneumonia cases in Wuhan, a city in the Hubei Province of China. It rapidly spread, resulting in a global pandemic. In February 2020, the World Health Organization named the disease COVID-19, which stands for coronavirus disease 2019. Its high transmission rate and the scarcity of effective treatment led to the rapid development of vaccines. By the end of 2020 , several vaccines had become available for use in different parts of the world, over 40 candidate vaccines were in human trials, and over 150 were in preclinical trials [1]. As a result, a series of adverse events after vaccination continue to be reported. Hereafter, we report a case of large vessel vasculitis (LVV) in patient who received the first dose of mRNA COVID-19 vaccine (BNT162b2-Pfizer-BioNTech). A 63-year-old woman with a personal history of hypertension has been observed at the end of June 2021 to a tertiarycare center (San Carlo Hospital), in Basilicata region (Italy). The day following the first vaccination (May 2021), she experienced fatigue, myalgias and after 5 days she rapidly developed fatigue as well as low grade fevers, anorexia, and headache. Over the next 4 weeks she complained a $15 \mathrm{lb}$ weight loss and polymyalgia rheumatica (PMR)- like symptoms (fatigue, arthralgia, stiffness of upper arms, shoulders and neck). She denied previous similar episodes or recent infectious diseases including COVID-19; a molecular diagnostic test for COVID-19 was also performed, and was negative. Physical examination showed limitation on the range of motion about the shoulders (inability to actively abduct the shoulders past 90 degrees) and the cervical

Michele Gilio

michelegilio@gmail.com

1 Infectious Diseases Unit, San Carlo Hospital, Potenza, Italy

2 Infectious Diseases Unit, Madonna Delle Grazie Hospital, Matera, Italy spine. Neurologic evaluation demonstrated normal muscle strength. Laboratory values were remarkable for elevated C-reactive protein $(74 \mathrm{mg} / \mathrm{L})$, erythrocyte sedimentation rate (ESR) $(104 \mathrm{~mm} / \mathrm{h})$, ferritin $(1227 \mathrm{ng} / \mathrm{mL})$ levels and mild normochromic, normocytic anaemia (haemoglobin $10.6 \mathrm{~g} /$ $\mathrm{dL}$ ). The following tests were negative or normal: antinuclear antibodies, antineutrophil cytoplasmic antibodies and cryoglobulins, C3, C4, serum thyroid-stimulating hormone (TSH), creatine kinase (CK), vitamin D and serology for hepatotropic viruses and HIV was negative. Positron emission tomography (PET) was performed and demonstrated an increased fluorodeoxyglucose (FDG) vascular uptake compatible with large-vessels vasculitis (Fig. 1). The distribution of vasculitis with large artery (aortic arch, thoracic and abdominal aorta) involvement of her carotid, subclavian arteries suggests Large-Vessel Vasculitis. A diagnosis of giant cell arteritis (GCA) would fit her onset of disease after age 50 and the subclavian changes, which may occur in GCA also in the absence of temporal artery involvement [2]. She refused contrast-enhanced MR angiography. According to the 2018 update of the EULAR recommendations for the management of large vessel vasculitis [3], for induction of remission, we initiated immediately with high dose glucocorticoid (GC) therapy ( $50 \mathrm{mg} /$ day prednisone-equivalent). On the fourth week systemic symptoms (anorexia, fever, malaise and arthralgias) resolved and CRP, ESR, ferritin and haemoglobin normalized. The tapering of GC therapy is ongoing. Up to now a systematic review revealed that influenza vaccine is the most often reported in post-vaccination cases of vasculitis [4]. The pathogenesis of postvaccination vasculitis remains unclear. To our knowledge, this is the first report of large vessel vasculitis following by BNT162b2 vaccination. Moreover, cases of vasculitis secondary to SARS-CoV-2 infection or after COVID-19 vaccination have also been reported in temporal association with their administration. (Table 1). Physicians should be aware of this complication, but should continue to encourage vaccination efforts given the well documented safety profile and efficacy of the Pfizer-BioNTech BNT16B2b2 mRNA vaccine [5]. Further randomized studies would be required 


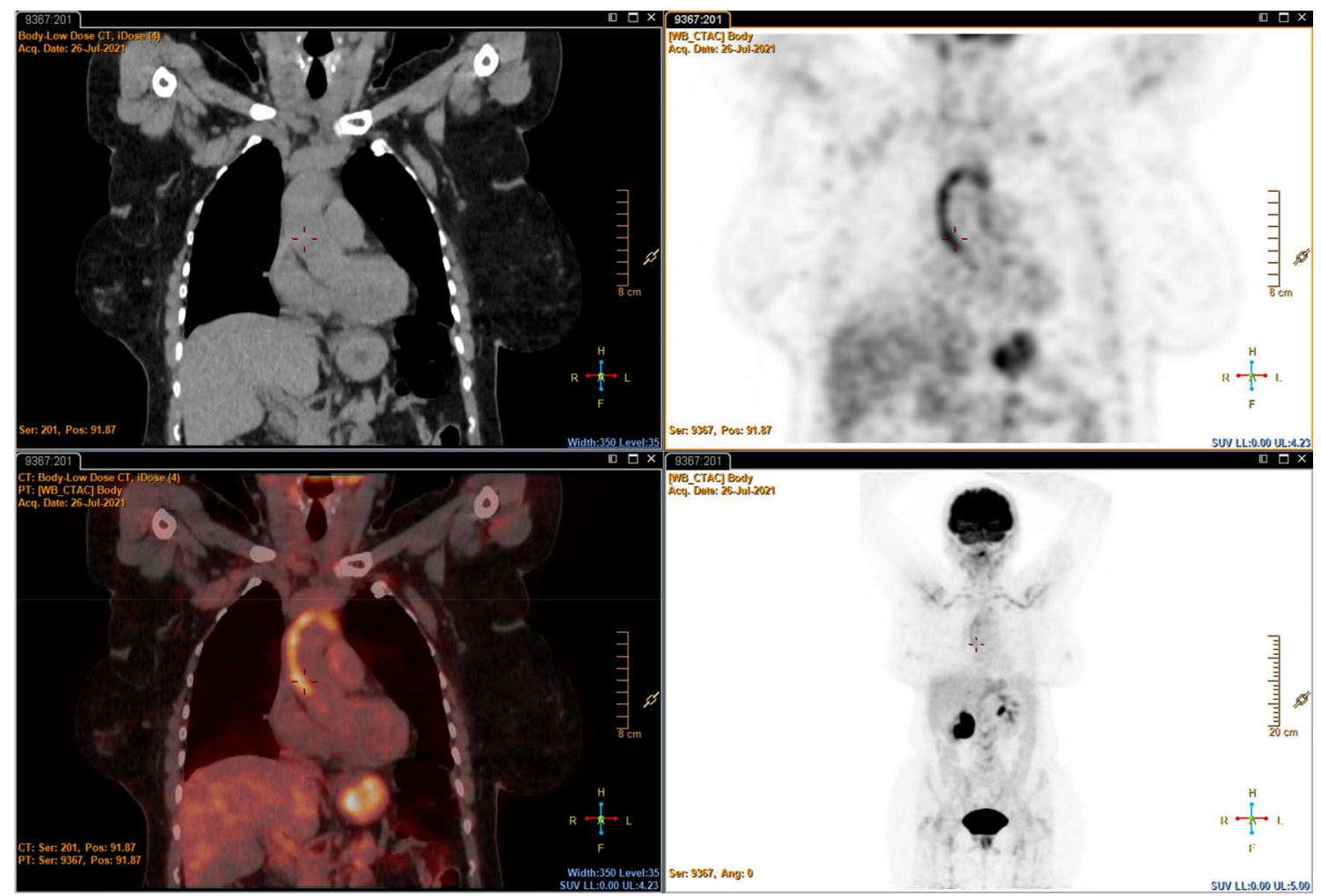

Fig. 1 Positron emission tomography (PET) that demonstrated an increased fluorodeoxyglucose (FDG) vascular uptake compatible with largevessels vasculitis

Table 1 Cases reported with vasculitis after COVID-19 vaccination

\begin{tabular}{lll}
\hline Author & Type of vaccine & Diagnosis \\
\hline $\begin{array}{l}\text { Dash et al. } \\
\text { Cohen et al. }\end{array}$ & adenoviral vector vaccine & Urticarial vasculitis \\
Bostan et al. & mRNA vaccine & Leukocytoclastic vasculitis \\
Shakoor et al. & mRNA vaccine & ANCA-associated vasculitis \\
Vassallo et al. & mRNA vaccine & Cutaneous lymphocytic vasculitis \\
Izzedine et al. & mRNA vaccine & Renal vasculitis \\
Obeid et al. & mRNA vaccine & Reactivation of IgA vasculitis \\
Nastro et al. & mRNA vaccine & Small vessel vasculitis \\
Gillion et al. & adenoviral vector vaccine & Granulomatous vasculitis \\
Hines et al. & mRNA vaccine & Henoch-Schönlein purpura \\
Guzmán-Pérez et al. & adenoviral vector vaccine & Small-vessel vasculitis \\
Berry et al. & adenoviral vector vaccine & Cutaneous small vessel vasculitis \\
\hline
\end{tabular}

to compare the frequencies and type of immune-mediated manifestations after these vaccines.

Acknowledgements We thank the medical and paramedical staff working in the Infectious Diseases Unit of the above-mentioned hospitals for constant and valuable support.

Author contributions We declare that all authors have made substantial contributions to this paper. In particular: MG contributed to the conception and design of the study, the acquisition of data, the analysis and interpretation of data, and contributed to the drafting the article; GDS revised critically the manuscript for important intellectual content. All authors gave final approval of the version to be submitted.

Funding We declare that no specific funds have been used for this paper. 


\section{Declarations}

Conflict of interest The authors declare that they have no competing interests.

Ethical approval This study was conducted according to the Declaration of Helsinkiand the guidelines of Good Clinical Practice. Written informed consent was obtained from this patient.

Human and animal rights All procedures performed were in accordance with the ethical standards of the institutional and/or national research committee.

\section{References}

1. World Health Organization. Draft landscape of COVID-19 candidate vaccines. https://www.who.int/publications/m/item/ draft-landscape-of-covid-19-candidate-vaccines. Accessed 20 Oct 2020

2. Brack A, Martinez-Taboada V, Stanson A et al (1999) Disease pattern in cranial and large vessel giant cell arteritis. Arthritis Rheum 42:311-317

3. Hellmich B, Agueda A, Monti S et al (2018) Update of the EULAR recommendations for the management of large vessel vasculitis. Ann Rheum Dis 2020(79):19-30

4. Bonetto C, Trotta F, Felicetti P et al (2016) Vasculitis as an adverse event following immunization-systematic literature review. Vaccine 34:6641-6651

5. Polack FP, Thomas SJ, Kitchin N et al (2020) Safety and efficacy of the BNT162b2 mRNA COVID-19 vaccine. N Engl J Med 383:2603-2615

Publisher's Note Springer Nature remains neutral with regard to jurisdictional claims in published maps and institutional affiliations. 